\title{
On Digital Therapeutics
}

\author{
Enrico Capobianco ${ }^{1,2 *}$ \\ ${ }^{1}$ Center for Computational Science, University of Miami, Miami, FL, USA, ${ }^{2}$ Laboratory of Integrative Systems Medicine, \\ Institute of Clinical Physiology, National Research Council, Pisa, Italy
}

Keywords: digital medicine, big data, social influence, collective sensing, Internet

Let us reconsider the criticism (Webb et al., 2010) that using the Internet to change people's behavior, through the delivery of targeted health interventions, lacks verification of effectiveness. In light of the growing social trend of person-generated health data, questions to consider are (1) What confidence should be assigned to "digital therapeutics"? and (2) How to start the dialogue with traditional therapeutics to acknowledge this trend?

\section{BACKGROUND}

The emerging crowdsourced health research has a largely unexplored potential. As part of a novel frontier of participatory health, it involves social networks, web-based studies, and smartphone applications. Examples include PatientsLikeMe with queries for conditions, symptoms, treatments, etc. ${ }^{1} ; 23 a n d M e$, dedicated to genetics ${ }^{2}$; MedHelp, focused on health communities, ${ }^{3}$ etc. These sites focus on drug response, user experiences, actions, and decisions reconstructing paths to endpoints, with or without clinician intervention. Other open spaces include the forum of Quantified Self ${ }^{4}$ or the crowdsourcing of Genomera, ${ }^{5}$ building individual and group activities centered on health-specific lifestyle (sleep, mood, diet, etc.).

Owing to a widely accessible lifestyle data domain, and a shift of personalized healthcare toward predictive tools and automated decision systems, Big Data in Health is destined to leverage tracking data generated by self-reports, mobile and other biosensing applications, publicly promoted diet and exercise programs, and information linked to social and sentiment analyses. The Internet-connected devices are clearly the new decision makers. Driven by complex intertwined signals (media, internet, etc.), these devices induce direct or mediated effects on synergistic communities. The physical and virtual dynamics enabled by collective sensors move beyond the limited individual perception of spatiotemporal events and phenomena; their attractors are behavior aggregation mechanisms centered on web listening and social reputation.

*Correspondence:

Enrico Capobianco

ecapobianco@med.miami.edu

\section{NEW IDEAS}

Specialty section:

This article was submitted to Big

Data, a section of the journal Frontiers in Digital Humanities

Received: 23 July 2015

Accepted: 26 October 2015

Published: 10 November 2015

Citation:

Capobianco E (2015)

On Digital Therapeutics.

Front. Digit. Humanit. 2:6. doi: 10.3389/fdigh.2015.00006

Collective sensing ${ }^{6}$ represents a sort of anthropic force enabling decisions and transmitting actions and then reconciling into average tendencies elaborated by social media. Clearly, sensing is exerted at the community level and depends only to a certain extent on measuring data volumes and replicated dynamics. Communities of any size, observable or hidden, may change for any reason

\footnotetext{
${ }^{1}$ http://www.patientslikeme.com/

${ }^{2}$ http://www.23andme.com/

${ }^{3}$ http://www.medhelp.org/

${ }^{4}$ http://quantifiedself.com/

${ }^{5}$ http://genomera.com/

${ }^{6} \mathrm{http}: / /$ www.collectivesensing.org/
} 
(time, interventions, etc.). Their influence is inherently determined by the participant nodes and their connectivities. The tasks of ensuring their detection and monitoring their evolution are crucial, but complicated by their dynamic nature. Therefore, conceiving new systems of measurement of social dynamics and collective events and elucidating their characteristics are urgent needs.

When enabling a scientific method, a factor to be assessed is the identification of real systematic patterns with significance criteria, thus creating consistency and reproducibility, together with biases, interferences, and confounders underlying such patterns. Big Data satisfies consistency, and less so reproducibility, as communities have specific identities, something hard to replicate. A key factor is determining the influence of the context in which the patterns are measured, in both spatial and temporal dimensions. Here, the anthropic principle could be called in, despite the controversy it brings (see the discussion following Prof. S. Hawking's Morris Loeb Lecture in Physics at Harvard University, in 1999, "Finite but Unbounded"7).

In medicine, there are signs of companion diagnostic, prognostic, preventive, and therapeutic decisions and actions emerging from Internet-driven digitalization, connectivity, and collective sensing dynamics. The most intriguing activity, digital therapeutics, is a product of behavioral changes induced by a variety of factors allowing people to make decisions that include self-assessment of medical results. The problem remains: How information on care, wellness, and therapy can support individual evaluations influenced by unknown dynamics (to some extent), and in part still difficult to quantify? What mediation processes are at play, and what possible impacts are exerted?

\section{SUPPORTING STUDIES}

Dorsch et al. (2015) focussed on the web-centered capacity of selfmonitoring chronic disease conditions awareness. The goal was improvement of quality of life in the case of chronic heart failure. The time frame for the prospective single-center and singlegroup study was 12 weeks, after which standard NYHA/MLHFQ examinations were repeated (baseline follow-up). The final classification in NYHA and the score in MLHFQ improved due to some factors (physical activity, diet, and physical examination). Self-management appeared as the key component, encouraging monitoring and prevention, self-adjustment, and interpretation of intermediate outcomes. Sepah et al. (2015) focused on a diabetes prevention program (Prevent) and performed web analyses with reference to participants followed for 2 years to measure effects on selected risk factors (body weight and Alc test). The participants underwent a 16 -week weight loss intervention program in combination with weight maintenance. The A1c test kits were monitored at 6 months, 1 year, and 2 years after the baseline start. Even if causal inference of the interventions to outcomes was not applicable, due to inherent study

${ }^{7}$ http://tech.mit.edu/V119/N48/47hawking.48n.html limitations (i.e., not externally controlled assignment of treatment), significant reductions in both risk factors were achieved at the end while maintaining weight loss in the intermediate times.

Peer-reviewed articles on Internet interventions appeared around the year 2000 (Ritterband and Tate, 2009). Open questions remain with regard to these interventions (classification, evaluation models, and cost-effectiveness analyses). New studies and evidences will appear in the near future from various medical contexts [see a review by Payne et al. (2015)]. With the possible consolidation of success stories, it is expected that marker identification, risk assessment, and clinical event prediction would need to be reevaluated. Self-monitoring and confidence effects at the individual level and sentiment influence and collective sensing at the crowd level are factors implying the intervention of drivers linked to mediation processes, social influence (Lorenz et al., 2011), and linkage, which spatiotemporally and contextually codetermined treatment outcomes. Notably, all such factors referring to the impacts from the exchange between health professionals and social media require in-depth analysis. Some of the impacts are quite easy to identify, for instance, creation of new spaces for interaction between patients and clinicians in support of disease management (Colera, 2013).

\section{CONCLUSION}

Recently, evidence is not just descriptive but also methodologically rigorous to support Internet-based digital therapeutics as verifiable and reproducible. By becoming more systematic (context-wise) and more systemic (patients outreach), such evidence-based approaches may contribute to change the assessment of human diseases, provide support to clinical decision making with novel collective data collection protocols and study designs, and motivate non-standard analyses inspired by Big Data.

It is commonly accepted that Big Data will yield a new disease taxonomy inspired by genotype-phenotype relationships, but will also go beyond symptoms and test data due to the integration of heterogeneous information. Nonetheless, treatment of biases and confounders, design of flexible clinical decision support systems (i.e., enabling automated covariate selection), and elaboration of new social network metrics (i.e., allowing sensitivity analyses and ad hoc propensity scores) are challenges destined to become either strengths or weaknesses of new scientific thinking.

\section{ACKNOWLEDGMENTS}

The author thanks two referees for providing useful comments. The author also thanks Prof. M. Lippman at the University of Miami for comments on the first drafts of the manuscript, his friend Dr. L. McVicker for reading it and improving the presentation style, and his friend Dr. N. Ianuale who introduced him to the anthropic principle during their recent philosophical discussion. 


\section{REFERENCES}

Colera, E. (2013). Social networks, social media, and social diseases. BMJ 346: f3007. doi:10.1136/bmj.f3007

Dorsch, M. P., Farris, K. B., Bleske, B. E., and Koelling, T. M. (2015). A web application for self-monitoring improves symptoms in chronic systolic heart failure. Telemed. J. E Health 21: 267-270. doi:10.1089/tmj.2014. 0095

Lorenz, J., Rauhut, H., Schweitzer, F., and Helbing, D. (2011). How social influence can undermine the wisdom of crowd effect. Proc. Natl. Acad. Sci. U.S.A. 108: 9020-9025. doi:10.1073/pnas.1008636108

Payne, H. E., Lister, C., West, J. H., and Bernhardt, J. M. (2015). Behavioral functionality of mobile apps in health interventions: a systematic review of the literature. J. Med. Internet Res. 3: e20. doi:10.2196/mhealth. 3335

Ritterband, L. M., and Tate, D. F. (2009). The science of Internet interventions. Ann. Behav. Med 38: 1-3. doi:10.1007/s12160-009-9132-5
Sepah, S. C., Jiang, L., and Peters, A. L. (2015). Long-term outcomes of a web-based diabetes prevention program: 2-year results of a single-arm longitudinal study. J. Med. Internet Res. 17: e92. doi:10.2196/jmir.4052

Webb, T. L., Joseph, J., Yardley, L., and Michie, S. (2010). Using the Internet to promote health behavior change: a systematic review and meta-analysis of the impact of theoretical basis, use of behavior change techniques, and mode of delivery on efficacy. J. Med. Internet Res. 12: e4. doi:10.2196/jmir.1376

Conflict of Interest Statement: The author declares that the research was conducted in the absence of any commercial or financial relationships that could be construed as a potential conflict of interest.

Copyright (c) 2015 Capobianco. This is an open-access article distributed under the terms of the Creative Commons Attribution License (CC BY). The use, distribution or reproduction in other forums is permitted, provided the original author(s) or licensor are credited and that the original publication in this journal is cited, in accordance with accepted academic practice. No use, distribution or reproduction is permitted which does not comply with these terms. 